\title{
Modélisation thermo-diffusionnelle de l'assemblage hétérogène acier/aluminium par mouillage réactif
}

\author{
Guillaume Sierra ${ }^{1,2, a}$, Cyril Bordreuil ${ }^{2}$, Patrice Peyre ${ }^{3}$, \\ Frédéric Deschaux-Beaume ${ }^{2}$, David Stuart ${ }^{3}$ et Gilles Fras ${ }^{2}$ \\ 1 CEA/DRT/LITEN/GERAILP, 16bis avenue Prieur de la Côte d'Or, 94114 Arcueil, France \\ 2 Laboratoire de Mécanique et Génie Civil, UMR 5508 CNRS, Université Montpellier II, CC 048 Place Eugène Bataillon, \\ 34095 Montpellier Cedex 5, France \\ 3 Laboratoire pour l'Application Industrielle des Lasers de Puissance, UPR 1578 CNRS, 16bis avenue Prieur de la Côte d'Or, \\ 94114 Arcueil, France
}

Reçu le 25 mai 2007, accepté le 24 janvier 2008

\begin{abstract}
Résumé - Le développement d'un modèle permettant la prédiction des dimensions de la couche de réaction formée lors de l'élaboration d'assemblages acier-aluminium par mouillage réactif TIG est présenté. La croissance de la phase $\mathrm{Fe}_{2} \mathrm{Al}_{5}$ formée à l'interface est régie par les transferts de chaleur au sein de la structure générés par l'apport d'énergie du procédé TIG. Une modélisation du procédé couplée avec la loi de croissance de la phase constituant la couche de réaction permet alors de prédire l'épaisseur et la largeur de cette couche.
\end{abstract}

Mots clés : Transfert de chaleur / diffusion / bi-matériaux / contact / intermétallique

\begin{abstract}
Thermo-diffusional modelling of dissimilar steel/aluminium assembly by reactive wetting. A numerical model allowing the prediction of the reaction layer size produced during the generation of steel-aluminium assemblies by TIG reactive wetting is developed. The $\mathrm{Fe}_{2} \mathrm{Al}_{5}$ growth produced along the interface is governed by the heat transfer in the structure caused by the welding energy deposed by the TIG process. A process modeling coupled with the growth law of the $\mathrm{Fe}_{2} \mathrm{Al}_{5}$ phase allows to predict the thickness and the width of the reaction layer.
\end{abstract}

Key words: Heat transfer / diffusion / bi-material / contact / intermetallics

\section{Introduction}

Afin de respecter les futures normes anti-pollution visant à réduire les émissions de $\mathrm{CO}_{2}$ dans l'atmosphère, les constructeurs automobiles envisagent de diminuer la masse des véhicules en remplaçant certains composants en acier par de l'aluminium. Pour atteindre ce but, il est nécessaire d'envisager des liaisons hétérogènes acieraluminium. C'est dans ce cadre qu'une étude sur l'assemblage entre des tôles d'acier galvanisé, ou non galvanisé et des tôles d'aluminium de $1 \mathrm{~mm}$ d'épaisseur a été réalisée par mouillage réactif (interaction entre acier solide et aluminium liquide) avec les procédés laser et TIG $[1,2]$. La tenue mécanique de ces assemblages est en grande partie liée aux dimensions (largeur et épaisseur) de la liaison intermétallique formée entre les deux matériaux.

\footnotetext{
a Auteur pour correspondance :

guillaume.sierra@gmail.com
}

Dans l'objectif d'optimiser la résistance de ces assemblages, le développement d'outils numériques permettant de modéliser la formation de la liaison hétérogène pourrait apporter de nouvelles solutions.

Pour l'assemblage par procédé TIG, une configuration d'assemblage par transparence est étudiée dans laquelle la fusion de l'aluminium est générée par conduction thermique à travers l'acier. Une couche de réaction intermétallique $\mathrm{Fe}_{2} \mathrm{Al}_{5}$ se développe suite à l'interaction entre l'acier solide et l'aluminium liquide, assurant ainsi la liaison entre les deux matériaux. L'étude de cette interaction en régime isotherme [3] a permis de conclure à la croissance diffusionnelle de la phase $\mathrm{Fe}_{2} \mathrm{Al}_{5}$ et de définir une loi de croissance isotherme.

En raison de la difficulté expérimentale à mesurer les cycles thermiques à l'interface acier-aluminium qui gouvernent la croissance des phases intermétalliques, on propose de développer dans une première étape une 
modélisation thermique du mouillage réactif TIG pour calculer ces cycles thermiques. Les cycles thermiques sont le résultat du transfert de chaleur généré par le procédé au sein de la structure. La configuration d'assemblage utilisée, ainsi que les différents éléments du montage influencent alors fortement l'allure du cycle thermique. Afin d'assurer la transférabilité des résultats à une configuration d'assemblage différente, il est nécessaire de modéliser les échanges de chaleur entre les différents éléments de la structure (bride, tôles...) par la prise en compte des conditions d'interaction thermique entre ces éléments. Au cours du soudage, il convient de prendre en compte l'évolution de la géométrie au cours de la création de la zone de réaction. Un modèle évolutif, reposant sur l'introduction de conductances thermiques de contact pour simuler les échanges de chaleur aux différentes interfaces sur des parties liées ou non liées est alors développé. Les résultats obtenus par la modélisation thermique sont validés par la mesure des cycles thermiques expérimentaux (thermocouples $\mathrm{K}$ ) et la dimension des zones fondues (micrographies).

Dans une seconde étape, les cycles thermiques calculés à l'interface acier-aluminium sont utilisés pour modéliser la croissance diffusionnelle anisotherme de la phase $\mathrm{Fe}_{2} \mathrm{Al}_{5}$. La diffusion étant un processus thermiquement activé, il est nécessaire de calculer pour chaque température de l'interaction la constante cinétique équivalente. Le modèle est alors validé par comparaison de l'épaisseur et de la largeur des couches de réaction calculées et mesurées expérimentalement.

\section{Croissance diffusionnelle de la phase $\mathrm{Fe}_{2} \mathrm{Al}_{5}$}

La mise en contact d'acier solide avec de l'aluminium liquide entraîne, en raison de la non-solubilité du fer dans l'aluminium à l'état solide la formation de phases intermétalliques $\mathrm{Fe}_{x} \mathrm{Al}_{y}$. L'étude des conditions de formation et de croissance de ces phases, réalisée dans le cadre de l'immersion d'acier solide dans de l'aluminium liquide pour des températures comprises entre $700{ }^{\circ} \mathrm{C}$ et $900{ }^{\circ} \mathrm{C}[3]$ a conclu à un schéma réactionnel en trois étapes, et la présence de trois mécanismes physiques : réaction chimique, diffusion et dissolution.

Durant la première étape, un contact s'établit entre les deux matériaux et se poursuit par une réaction chimique aboutissant à la formation d'une couche limite de $\mathrm{Fe}_{2} \mathrm{Al}_{5}$. La croissance de cette phase se poursuit par un processus de diffusion, ce qui constitue la deuxième étape. La troisième phase intervient avec l'apparition d'un nouveau mécanisme, simultané à la croissance diffusionnelle de la couche de réaction, qui correspond à un phénomène de dissolution des phases intermétalliques formées dans l'aluminium liquide.

Suivant ce modèle les auteurs [3] établissent alors une loi de croissance parabolique en régime isotherme (1) basé sur le phénomène de diffusion :

$$
x=\sqrt{2 k t}
$$

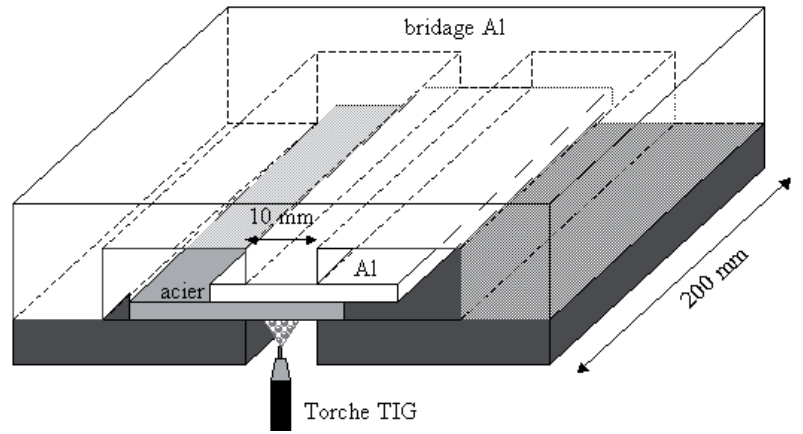

Fig. 1. Configuration d'assemblage acier-aluminium par mouillage réactif TIG.

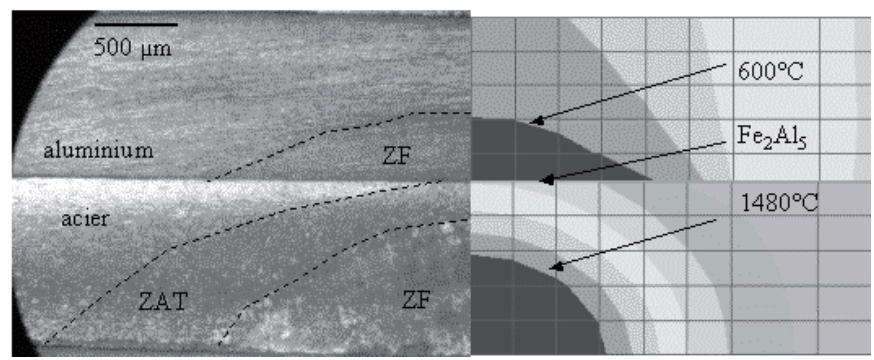

Fig. 2. Zone de liaison expérimentale et simulée.

avec $x$ : épaisseur de la couche $(\mathrm{m}), t$ : temps d'interaction (s) et $k$ : constante cinétique de croissance $\left(\mathrm{m}^{2} \cdot \mathrm{s}^{-1}\right)$.

La croissance de ces couches de réaction n'induit pas de modification du régime thermique au sein de l'assemblage. Dans le modèle numérique, on prendra uniquement en compte la présence, ou non, d'une couche de réaction.

\section{Conditions expérimentales du mouillage réactif acier-aluminium}

L'assemblage entre des tôles $(200 \mathrm{~mm} \times 60 \mathrm{~mm} \times$ $1 \mathrm{~mm})$ d'acier bas carbone DC $04 \quad(0,08 \quad \% \quad \mathrm{~m}$. $\mathrm{C} ; 0,4 \% \mathrm{~m}$. Mn) et d'alliage d'aluminium 6016-T4 $(0,4 \%$ m. Mg; $1,1 \%$ m. Si) est étudié par le procédé TIG (Tungsten Inert Gas). La fusion partielle de l'acier placé en position inférieure (Fig. 1), réalisée par soudage au plafond entraîne la fusion de l'aluminium par conduction thermique à travers l'épaisseur de la tôle.

La formation et la croissance de la phase $\mathrm{Fe}_{2} \mathrm{Al}_{5}$ se déroule ensuite dans le plan de joint entre l'acier solide et l'aluminium liquide (Fig. 2).

Afin de valider les modélisations thermiques du procédé, les essais expérimentaux sont instrumentés thermiquement avec des thermocouples $\mathrm{K}$ gainés (diamètre $0,5 \mathrm{~mm}$ et $1 \mathrm{~mm}$ ) logés dans des trous de diamètre identique (Fig. 3) en différents points de la structure afin d'obtenir les cycles thermiques. La dimension des zones fondues (ZF) et des zones affectées thermiquement (ZAT) de l'acier et de l'aluminium est obtenue expérimentalement par analyse micrographique de coupes transverses (Fig. 2) 
Tableau 1. Valeurs des conductances thermiques d'interface utilisées pour le calcul thermique $[1,6]$.

\begin{tabular}{|l|l|}
\hline Nature du contact & Conductance thermique $\left(\mathrm{W} \cdot \mathrm{m}^{-2} \cdot \mathrm{K}^{-1}\right)$ \\
\hline Acier solide/acier solide & $10^{3}$ \\
\hline Acier solide/aluminium liquide & $1,5 \times 10^{5}$ \\
\hline Aluminium solide/aluminium solide & $5 \times 10^{3}$ \\
\hline
\end{tabular}

(a)

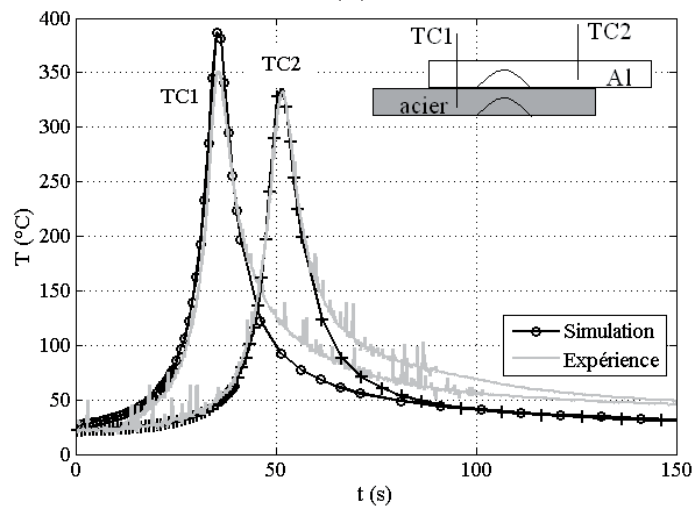

Fig. 3. Cycles thermiques expérimentaux et numériques, TC : thermocouple $\mathrm{K}$.

Les cycles thermiques relevés en différents points de la structure (Fig. 3) montrent une diminution des températures maximales lorsque l'on s'éloigne de la source de chaleur, depuis l'acier vers la bride. Les cycles thermiques vus à l'interface acier-aluminium sont donc fortement influencés par le transfert de chaleur au sein de la structure, et donc par la configuration d'assemblage.

Le calcul des cycles thermiques interfaciaux nécessaire au calcul diffusionnel de la croissance de $\mathrm{Fe}_{2} \mathrm{Al}_{5}$ impose alors de modéliser l'ensemble du montage expérimental et de prendre en compte les échanges thermiques et la conduction dans l'ensemble des composants de la structure.

\section{Modélisation thermique}

La modélisation thermique est réalisée sous SYSWELD ${ }^{\circledR}[4]$ en utilisant les dimensions réelles de l'assemblage et un modèle tridimensionnel. Les paramètres dimensionnels de la source Goldak [5] classiquement utilisée pour la modélisation du soudage à l'arc sont identifiés à partir de coupes micrographiques obtenues après soudage (Fig. 2).

Les conditions aux limites réalistes imposées au modèle permettent de prendre en compte les échanges de chaleur avec le milieu extérieur par convection $(h=$ $\left.15 \mathrm{~W} \cdot \mathrm{m}^{-2} \cdot \mathrm{K}^{-1}\right)$ et rayonnement $(\varepsilon=0,5)$. Afin de modéliser le transfert de chaleur aux différentes interfaces, on introduit des conductances de contact [6] en fonction de la nature de l'interaction (solide/liquide ou solide/solide) et de la nature des matériaux (acier/Al ou
$\mathrm{Al} / \mathrm{Al})$. Les valeurs de conductance thermique d'interface utilisées pour le calcul thermique sont rassemblées dans le tableau 1.

$$
Q=h A \Delta T
$$

avec $h$ : conductance thermique $\left(\mathrm{W} \cdot \mathrm{m}^{-2} \cdot \mathrm{K}^{-1}\right), A$ : aire de contact $\left(\mathrm{m}^{2}\right)$ et $\Delta T$ : écart de température entre les deux surfaces $(\mathrm{K})$, où la différence de température est celle de deux nœuds en vis-à-vis.

La conductance thermique varie avec la nature des matériaux mis en contact (rugosité, dureté, conductivité thermique) et avec la pression appliquée. Le modèle développé dans cette étude considère uniquement une conductance constante fonction de l'interaction et ne prend pas en compte la pression de contact.

Afin de rendre compte de l'évolution au cours du soudage des différentes zones, il convient de définir une gestion évolutive des conductances thermiques. Une fois la couche de réaction créée, un bon transfert de chaleur sera établi à travers la zone de liaison alors qu'un contact thermo-mécanique entre les deux tôles d'acier et d'aluminium transfèrera moins bien la chaleur. En séparant les zones liées des zones non liées, on peut donc affecter une conductance thermique différente. Afin de prédire la zone de liaison entre les deux corps, il faut donc établir un critère qui définisse la formation d'une zone liée entre l'acier et l'aluminium en chaque point de la structure. Le critère le plus simple peut être défini à partir de la température de fusion de l'aluminium (et pas l'acier), ce qui se traduit par la formation d'une couche de réaction. Lorsque la liaison est établie les deux points situés de part et d'autre de l'interface ont la même température.

Du point de vue numérique, la gestion des zones liées nécessite le transfert entre différents groupes et doit donc être évolutive. Une zone pour laquelle il y a contact mécanique initial et qui voit la formation d'une couche de réaction devra être transférée vers une zone de liaison.

Chacune de ces deux zones a une conductance thermique différente. Pour une zone de contact mécanique, la conductance évolue en fonction de la température à la chauffe et au refroidissement, alors que pour la zone de liaison, la conductance thermique doit être maintenue à un niveau de conductance élevé afin d'imposer les températures égales de part et d'autre de l'interface.

À l'état initial, tous les nouds sont affectés à la zone de contact mécanique. Au fur et à mesure du calcul, des zones deviennent liées et il convient alors de modifier leur appartenance à une zone. Le passage d'une zone à l'autre est gouverné par le critère défini précédemment.

La procédure de modification des caractéristiques pour un point passant d'une zone en contact mécanique à 
(a)

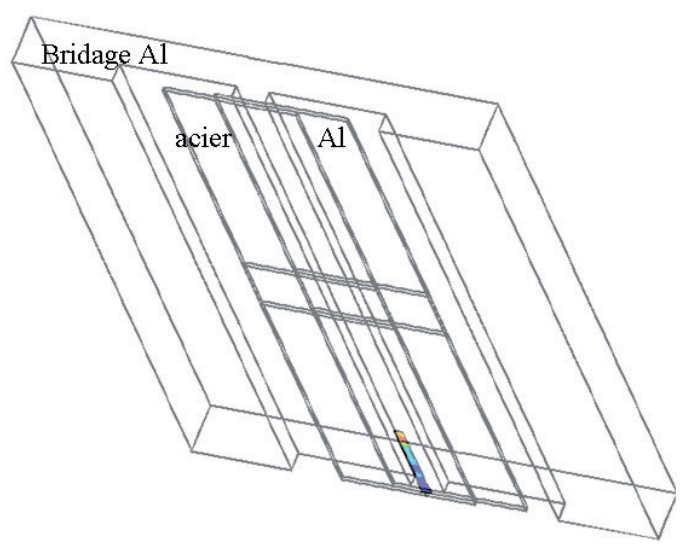

(c)

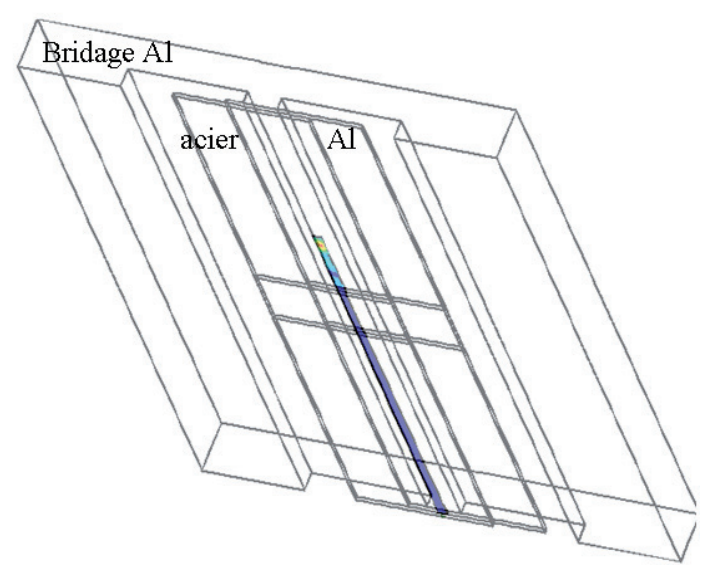

(b)

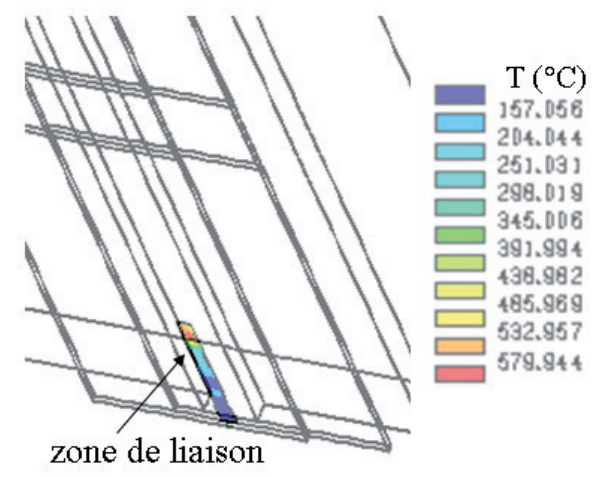

(d)

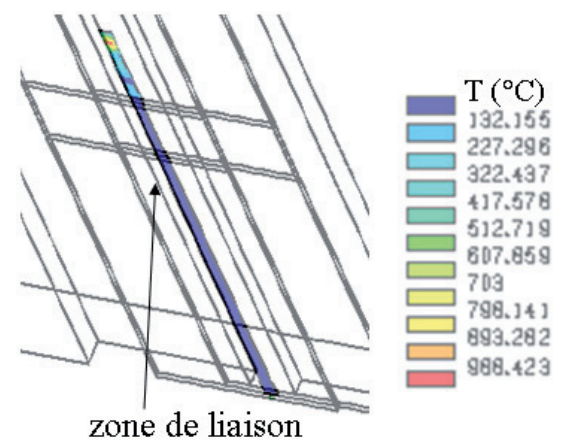

Fig. 4. Zone de liaison à $t=10 \mathrm{~s}$, (a) vue d'ensemble et (b) grossissement de la zone de liaison. Zone de liaison à $t=65 \mathrm{~s}$, (c) vue d'ensemble et (d) grossissement de la zone de liaison.

une zone de liaison induit le choix de l'instant de calcul et du suivi de la zone de liaison. Ces deux problèmes peuvent induire des problèmes de convergence de la méthode numérique. On décide de faire la modification une fois l'incrément de calcul effectué et non au cours du calcul pour des raisons de simplicité. La modification de la conductance thermique est réalisée lorsque la température dans l'aluminium a atteint la température de fusion, une fois la zone liée, on maintient ce niveau de conductance élevé. Dans ce cas, les modifications du type de zone n'induisent pas de discontinuité au niveau du calcul puisque la conductance est la même à cet instant pour la zone en contact et la zone liée.

Pour gérer de manière efficace la modification des zones, il convient de faire dialoguer différentes entités introduites dans le modèle. La conductance est une caractéristique de l'interface juxtaposée aux éléments de contact. Le critère établissant la formation d'une zone de liaison est défini sur les nœuds de l'interface du côté de la tôle d'aluminium. Il convient alors de gérer une base de données faisant dialoguer les éléments d'interface avec les nœuds. La base de données est évolutive et doit être réactualisée à chaque pas de temps, de plus elle est modifiée chaque fois que le critère est atteint.
On transfère alors tous les éléments voisins des nœuds concernés d'un groupe vers l'autre. Cette manipulation permet de déterminer la taille de la zone de liaison de manière explicite, elle est déterminée a posteriori en fonction du régime thermique. Les limites de cette approche résident dans le fait que la prédiction de la zone de liaison est dépendante du maillage. En effet, les éléments sont transférés lorsque le critère est atteint en chaque nœud. Cette approche nécessite donc d'utiliser un maillage relativement fin.

L'évolution de la zone de liaison au cours du soudage est présentée en figure 4.

On remarque sur cette figure, l'évolution de la création de la zone de liaison au cours du temps. La largeur de la couche de réaction reste constante entre $10 \mathrm{~s}$ (Figs. 4a et $\mathrm{b}$ ) et $65 \mathrm{~s}$ (Figs. 4c et d), ce qui traduit le régime quasi-stationnaire du procédé.

Le modèle numérique est ensuite validé par comparaison des tailles des zones de fusion (acier et aluminium) et des cycles thermiques mesurés en différents points de la structure.

Une fois les cycles thermiques interfaciaux calculés et extraits du calcul, ils peuvent être introduits dans le calcul diffusionnel de la croissance de la phase $\mathrm{Fe}_{2} \mathrm{Al}_{5}$. 


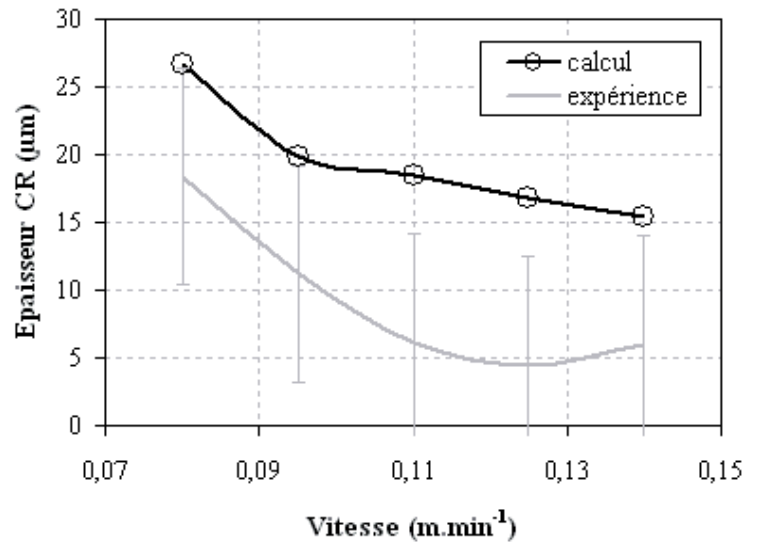

Fig. 5. Épaisseur de la couche de réaction (CR) $\mathrm{Fe}_{2} \mathrm{Al}_{5}$ calculée et mesurée expérimentalement.

\section{Loi de croissance diffusionnelle}

L'adaptation des lois de croissance isotherme de la phase $\mathrm{Fe}_{2} \mathrm{Al}_{5}$ au régime anisotherme rencontré en soudage est réalisé par le calcul de la constante cinétique pour chaque température via la loi d'Arrhénius (3).

$$
k_{T}=k_{0}\left(-\frac{Q}{R T}\right)
$$

avec $k_{T}$ : constante cinétique à la température $T$ $\left(\mathrm{m}^{-2} \cdot \mathrm{s}^{-1}\right), k_{0}$ : facteur de fréquence, $Q$ : énergie d'activation apparente $\left(\mathrm{J} \cdot \mathrm{mol}^{-1}\right), T$ : température $(\mathrm{K})$ et $R$ : constante des gaz parfaits $\left(R=8,314 \mathrm{~J} \cdot \mathrm{mol}^{-1} \cdot \mathrm{K}^{-1}\right)$.

La variation de la constante cinétique $k_{T}$ en fonction de la température nécessite une résolution différentielle de l'équation de croissance (1).

Les valeurs de l'énergie d'activation $(Q=$

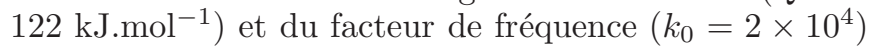
sont moyennées à partir des différentes données bibliographiques [3]. Les cycles thermiques interfaciaux calculés précédemment sont alors utilisés pour calculer l'épaisseur (Fig. 5) et la largeur de la couche de réaction $\mathrm{Fe}_{2} \mathrm{Al}_{5}$ formée lors du mouillage réactif acier-aluminium.

L'ordre de grandeur des résultats est très satisfaisant ainsi que les tendances observées. Ce type de calcul permet alors d'optimiser les paramètres expérimentaux du procédé et en particulier le type de conditions aux limites pour optimiser l'épaisseur des intermétalliques.
Le modèle thermo-diffusionnel ainsi développé permet donc de modéliser avec un bon accord expérience/simulation la croissance de la phase $\mathrm{Fe}_{2} \mathrm{Al}_{5}$ à partir des paramètres de soudage et de la prise en compte de la configuration d'assemblage et des conditions d'échanges thermiques interfaciaux par l'introduction de conductance thermique de contact.

\section{Conclusion}

Le modèle numérique développé dans ce travail permet donc d'établir un passage direct des paramètres expérimentaux de soudage à la morphologie (épaisseur et largeur) de la couche de réaction développée lors de l'interaction acier solide-aluminium liquide, grâce à l'intégration d'une analyse thermo-diffusionelle dans le calcul. La prise en compte de la configuration d'assemblage (dimensions réelles) et des conditions aux limites (échanges avec le milieu extérieur et contact thermique aux interfaces) permet donc d'envisager le transfert de ce modèle à d'autres configurations d'assemblage afin d'optimiser, soit la géométrie de la configuration, ou de l'assemblage, soit les paramètres de soudage à partir d'un critère défini au préalable. Par exemple l'optimisation de la tenue mécanique de ces liaisons rendue possible par l'augmentation de la largeur des couches de réaction [1] pourrait être réalisée par des essais numériques.

\section{Références}

[1] G. Sierra, Étude métallurgique et mécanique de l'assemblage hétérogène acier/aluminium par les procédés laser et TIG, Thèse, Université Montpellier II, 2006

[2] P. Peyre, G. Sierra, F. Deschaux-Beaume, D. Stuart, G. Fras, Generation of aluminium-steel joints with laserinduced reactive wetting, Mater. Sci. Eng. A 444 (2007) 327-338

[3] A. Bouayad, C. Gerometta, A. Belkebir, A. Ambari, Kinetic interactions between solid iron and molten aluminium, Mater. Sci. Eng. A 363 (2003) 53-61

[4] Manuel de reference, SYSWELD 2000, ESI GROUP, 2000

[5] J. Goldak, A. Chakravarti, M. Bibby, A new finite element model for welding heat sources, Metal. Trans. B 15B (1984) 299-305

[6] C.V. Madhusudana, Thermal contact conductance, Springer, 1996 